\title{
The role of embodied cognition in linguistic mental health analysis
}

\author{
$\underline{\text { D. Kernot }}^{\text {a }}$, M. Van Hooff ${ }^{\text {bc }}$, E. Lawrence-Wood ${ }^{\text {bd }}$, L. Glenny ${ }^{\mathrm{e}}$, C. Snowden ${ }^{\mathrm{e}}$, K. Green $^{\mathrm{e}}$ and C. Son $^{\mathrm{e}}$ \\ ${ }^{a}$ Defence Science and Technology Group, Edinburgh, SA, Australia, ${ }^{b}$ Centre for Traumatic Stress Studies, \\ University of Adelaide, Adelaide, SA, Australia, ${ }^{c}$ Military and Emergency Services Health Australia, \\ Glenside, SA, Australia, ${ }^{d}$ Phoenix Australia Centre for Posttraumatic Mental Health, University of \\ Melbourne, Melbourne, Victoria, Australia, ${ }^{e}$ University of South Australia, Magill, SA, Australia. \\ Email: david.kernot@dst.defence.gov.au
}

\begin{abstract}
Drawing on the intersection between embodied cognition and the referential process, where the human body and its environment influence the way a person thinks and feels, we compared two linguistic analysis approaches to see if it was possible to classify self-reported mental health in female veterans from their writing using words affected by the emotional and modality-specific systems in the brain. Drawing on self-reported PTSD, Depression, and psychological distress properties in the Diagnostic and Statistical Manual of Mental Disorders (DSM-5) we used interview transcripts $(n=40)$ and compared them to normal bloggers' posts $(n=25)$ and discovered statistically significant differences in language use (total of 330,000 words). We used two linguistic approaches, the Linguistic Inquiry Word Count (LIWC) tool, and an embodied cognition approach (Richness, Personal Pronouns, Referential Activity Power, and Sensory (RPAS) algorithm) to statistically correlate findings. In this exploratory study, findings suggested that deployed female military personnel have a distinct pattern of speech which is more blunted (use fewer unique words but more repetition) than women in the community. This style of language is often seen in individuals with anxiety and depression. Deployment length $(2-20$ months, average $=8.5)$ directly correlated to increased blunted speech and was related to an increased feeling of emotional numbness and greater un-conscious reliance on memories when generating language. There were also language differences in the female military cohort who self-report more mental health symptoms. Those with self-reported mental health conditions $(n=7)$ used words that focussed on the past and used fewer 'they' type words and isolated themselves to minimise stigma. This stigmatised language implied female military deployees with mental health symptoms felt isolated from their peers, through 'othering' and members of an outgroup. This study supports the idea that linguistic analysis using embodied cognition can discriminate between military females with self-reported mental health and those without and may be a valuable aid in highlighting post-traumatic stress in veterans.
\end{abstract}

Keywords: Mental health, post-traumatic stress, embodied cognition, LIWC 


\section{INTRODUCTION}

Historically, cognition was understood as the result of processes occurring solely in the brain, however, cognitive scientists studying embodied cognition have begun emphasising the role of the body and environment in which the brain is an "open system" (Hotton \& Yoshimi, 2010). All cognition is fundamentally embodied (Kernot, 2019), that is, closely linked to the motor and sensory surfaces, strongly dependent on experience, on context, and on being immersed in rich and structured environments. (Schöner \& Reimann, 2019). Intelligence emerges in the interaction of an organism with an environment and as a result of sensory-motor activity (Smith, 2005).

The core of cognition forms around such things as language, thought, reasoning, or knowledge (Schöner \& Reimann, 2019). Each person carries in their head a mental model, an abstraction of all their perceptions and experiences from real-world events, which they use to guide their decisions and has been vitally important to the field of system dynamics since its inception in Industrial Dynamics (see Forrester, 1971; 1997 in Doyle \& Ford, 1998). Cognition is detached from the motor and sensory surfaces and has given rise to the theoretical framework of information processing in which cognition is the manipulation of symbolic information (Schöner \& Reimann, 2019). If cognition can span the brain, body, and the environment, then it is an extended system assembled from a broad array of resources (Wilson \& Golonka, 2013). While dynamical systems are traditionally viewed as "closed systems", by extending the framework of dynamical systems theory, structures which appear in the state space of an embodied agent differ from those that appear in "closed systems" and can model representational processes in embodied agents (Hotton \& Yoshimi, 2010).

Cognition is in many ways an emergent phenomenon and one of the strengths of the dynamical approach to cognition is its ability to describe the emergence of complex structures and processes which are a computational representation of the mental computer, taking symbolic representations as inputs and computing symbolic representations as outputs where periphery transducers transform sensory stimulation into input representations and output representations into physical movement of the body (Port, \& Van Gelder, 1995). Embodied and dynamical system approaches to cognition have sometimes been presented as radical alternatives to traditional cognitive science, and as a means of overcoming the use of such traditional constructs as internal computational structures and representations, which mediate between sensory input and behavioural output, but by extending dynamical systems theory, traditional cognitive models can be incorporated into the study of embodied cognition (Hotton \& Yoshimi, 2011). Here, we highlight two computational classifiers that support a systems dynamics approach to model mental health through embodied cognition.

This exploratory study seeks to classify mental health in female veterans from their writing style. It creates a linguistic framework that uncovers blunted and stigmatising language with a focus on the past. These psycholinguistic properties can be used to highlight mental health concerns as highlighted by the following.

\subsection{Two Linguistic classifiers}

Every emotional state is generally accompanied by the retrieval of specific images and events relevant to that state. In order to put these emotional states or experiences into words, connections are made with non-verbal and non-discrete, analogic, or sub-symbolic information in the mind through what is termed the referential process and these connections translation of non-verbal dominant emotional schemas into logical, organised speech (Bucci \& Miller, 1993; Murphy, Maskit \& Bucci, 2015). We use two unique linguistic programs to classify language:

The Linguistic Inquiry and Word Count (LIWC) program calculates the proportion of words in a text that refers to emotions and ways of thinking, as well as analysing speech patterns and references to social issues. By analysing this data, the program aims to identify, through language, an individual's social and emotional state (Pennebaker et al., 2015; Pennebaker Conglomerates Inc., 2015). LIWC was originally developed to identify links between language use, such as content and style words, and psychological constructs, such as emotion and cognition (Tausczik \& Pennebaker, 2010). LIWC use in other research projects has revealed a number of findings of relevance to this study, providing a well-documented linguistic framework of the psychometric properties of a person through language. For example, it has been used to develop classifiers that can identify increased rates of PTSD among US military base populations, based on the characteristics of the language these individuals used (Coppersmith, Harman, \& Dredze, 2014). LIWC has also detected an inverse correlation between the use of cognitive words and post-treatment anxiety, as well as a negative relationship between social adjustment and the use of death words and negative emotion words (Alvarez-Conrad, Zoellner, \& Foa, 2001). Additionally, Papini et al's (2015) study examined language use of two groups of trauma-exposed participants - one group with PTSD, and one without. The study found that participants with PTSD used more third-person singular pronouns and death-related words, and fewer third-person plural pronouns. Participants 
with PTSD also exhibited a non-significant trend towards increased use of sad-related words, but there was no significant difference in the use of anxiety words or cognitive flexibility words between the two groups.

The Richness, Personal Pronouns, Referential Activity Power, and Sensory (RPAS) algorithm or classifier (Kernot, 2019) provides a signature of a person's writing based on personality markers. By examining these individual reference points of text over time, it is possible to see change in an individual that may indicate posttraumatic stress disorder (PTSD), anxiety and depression, or cognitive decline, as biomarkers of personality. This classifier provides a complementary psycholinguistic model of a person's cognitive state around the embodied cognition. These lived experiences and emotions can highlight trauma and mental health issues in a person's language. Signs of 'othering', perceiving and treating someone as inferior to yourself or the group to which you belong, can also be identified.

\section{METHODS}

We used LIWC and RPAS classification techniques to construct a psycholinguistic model representative of an individual's underlying cognitive state. Data from 40 transcribed interviews (Lawrence-Wood et al., 2014), a sample of full-time employed mothers who had deployed to the Middle East Area of Operations (MEAO) and PCL-5 (PTSD), PHQ-9 (Depression) K10 (psychological distress) all associated self-reported mental health questionnaire data from the Diagnostic and Statistical Manual of Mental Disorders, DSM-5 (American Psychiatric Association, 2013) was used to identify characteristics of mental health in language (total words 195,557 , unique words 29,727). We correlated the women veteran's length of deployment using Pearson's Correlation coefficient in order to examine the relationship between length of service and cognitive processing as indicated by the Richness of the language used by participants, and their Referential Activity Power values.

An independent, anonymised sample from publicly available web blogs about women's support group topics was also collected from women who did not identify as being military or having deployed to a war zone. This data was broken into 25 posts (total words 134,473, unique words 30,309 ), so that the average was the same as the women veterans' data. Data was also structured to ensure that the minimum and maximum file size was the same as the minimum and maximum file size of the women veterans' data.

Using the results from LIWC and RPAS, the psychometric properties of the language from both datasets were then compared using an independent-samples $t$-test in order to determine if serving in the military (and being on an operational deployment) changes the linguistic characteristics of deployed women. These same psychometric properties were then compared using an independent-samples $t$-test in order to determine if any differences in the characteristics between those deployed women who self-reported mental health concerns and those deployed women who did not self-report mental health concerns. LIWC was used to examine the differences in the emotional content of the language used by both groups of women. LIWC was used to examine differences in "othering" through the use of singular pronouns such as 'I,' 'me,' and 'my' (Nook, Schleider, \& Somerville, 2017).

\section{RESULTS}

There was a statistically significant but moderate relationship between the length of service conducted in weeks and Referential Activity Power $(r=.421 \mathrm{p}=.016$ at the 0.05 level (2-tailed) $\mathrm{n}=32)$. There was also a significant inverse linear relationship between Richness and Referential Activity Power $(r=-.416 p=.008$ at the 0.01 level (2-tailed) $n=40$ ) which is referred to as Cognitive Processing. Deployed military service length was converted to weeks and a set of Pearson correlations were computed on the military women sample to determine significant relationships between psycholinguistic variables and length of deployed military service. Cognitive processing was indicated by participants' language Richness and Referential Activity Power values.

We found the mean Richness score of Mothers who served in the MEAO $(M=0.166882$, sd $=0.037233)$ was statistically significantly different $(\mathrm{t}=-6.815, \mathrm{df}=41.248$, two-tailed $\mathrm{p}=.000)$ from women writing in selfhelp groups $(\mathrm{M}=0.24461, \mathrm{sd}=0.048847)$. The mean Referential Activity Power score of Mothers who served in the MEAO $(\mathrm{M}=0.188637, \mathrm{sd}=0.00603)$ was statistically significantly different $(\mathrm{t}=8.466, \mathrm{df}=35.964$, two-tailed $\mathrm{p}=.000)$ from women writing in self-help groups $(\mathrm{M}=0.170497, \mathrm{sd}=0.009595)$. Drawing on Levine's Test for Equality of Variances, the variances for the two groups were significantly unequal ( $\mathrm{F}=0.578$, $\mathrm{p}=0.45$ ).

The mean Sensory component score of Mothers who served in the MEAO $(\mathrm{M}=0.005737$, $\mathrm{sd}=0.001619)$ was statistically significantly different $(t=-7.656, \mathrm{df}=309.01$, two-tailed $\mathrm{p}=.000)$ from that of women writing in self-help groups $(\mathrm{M}=0.011651$, sd $=0.003644)$. Drawing on Levine's Test for Equality of Variances, highlighted the variances for the two groups were significantly unequal $(F=13.991, p=0.000)$ and the output line for unequal variances was used. 
Through an independent-samples $t$-test to evaluate if deployed military mothers' psycholinguistic signatures were statistically significantly different from a non-military women's sample who posted in self-help groups. The lower Richness of the MEAO Women indicated less unique word use, or more repetition in speech, and an indicator of anxiety and depression in individuals. While the higher Referential Activity Power and lower Sensory component across all of the sensory modalities mean values for MEAO Mothers highlighted highly concrete and high in imageability visual, auditory, haptic, olfactory and gustatory words, which suggested more concrete language relying highly on stored imagery. These same psychometric properties were then compared using an independent-samples $t$-test in order to determine if any differences existed in the linguistic characteristics between those deployed women who self-reported mental health concerns and those deployed women who did not self-report mental health concerns.

We found four statistically significant differences in 'They' words, articles, focussing on the past, and with sensory auditory words. In Figure 1, the mean total 'they' word score of those with low self-reported PTSD $(\mathrm{M}=1.7, \mathrm{sd}=.585)$ was statistically significantly different $(\mathrm{t}=2.836, \mathrm{df}=38$, two-tailed $\mathrm{p}=.007)$ from that of people with high scores of self-reported PTSD $(\mathrm{M}=1.05, \mathrm{sd}=.343)$. The effect size of Hedges' $\mathrm{g}=1.18$ implies a very large effect. As seen in Figure 2, the mean total 'focuspast' word score of those with low selfreported PTSD $(\mathrm{M}=7.28, \mathrm{sd}=1.21)$ was statistically significantly different $(\mathrm{t}=-2.538, \mathrm{df}=38$, two-tailed $\mathrm{p}$ $=.015)$ from that of people with high scores of self-reported PTSD $(\mathrm{M}=8.54, \mathrm{sd}=1.1)$. The effect size of Hedges' $\mathrm{g}=1.06$ implies a very large effect.
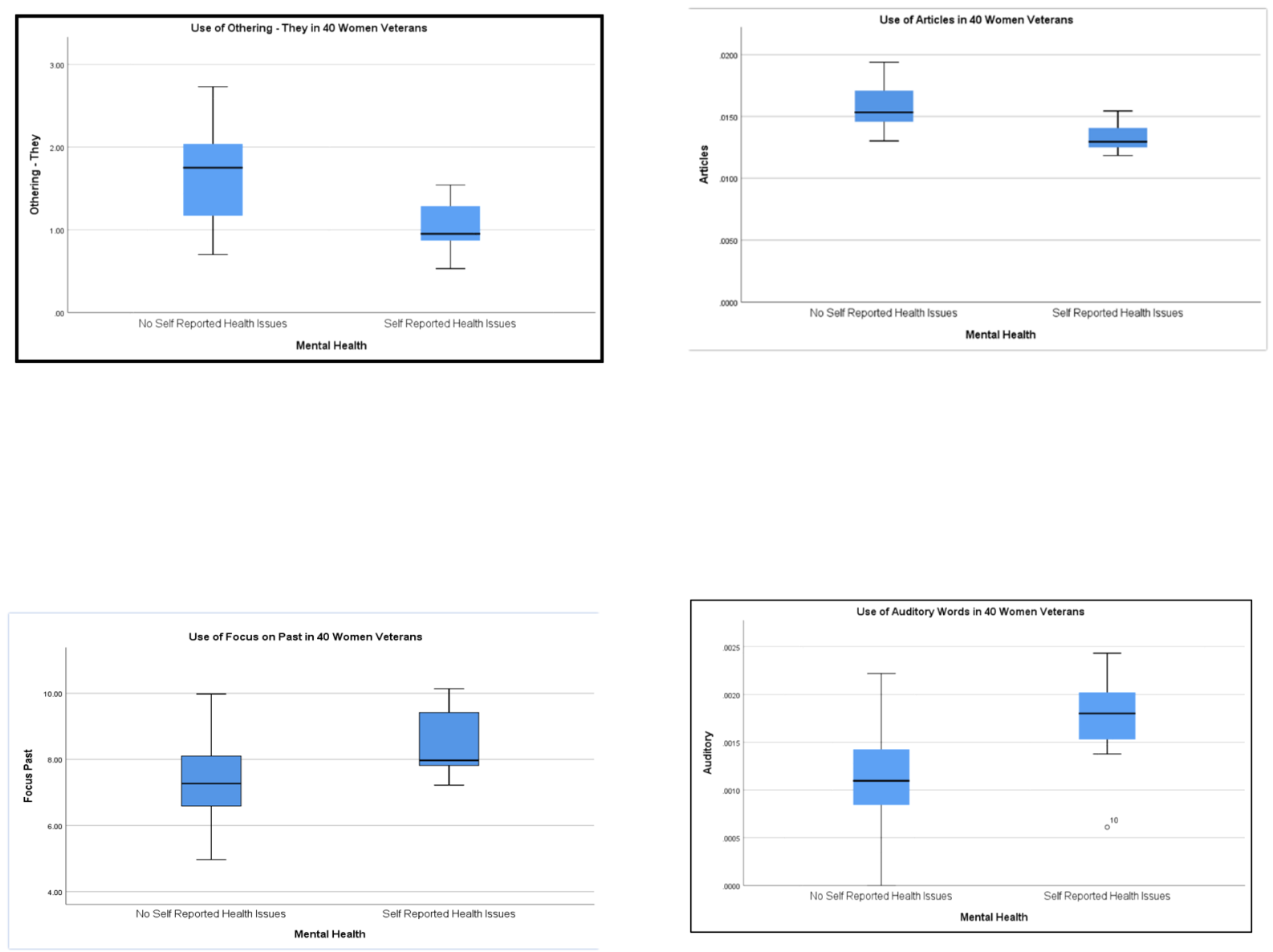
Figure 3 above highlights the mean total Articles (An) score of those with low self-reported PTSD (M $=.016$, sd $=.0015)$ was statistically significantly different $(\mathrm{t}=3.677, \mathrm{df}=38$, two-tailed $\mathrm{p}=.001)$ from that of people with high scores of self-reported PTSD $(\mathrm{M}=.0133, \mathrm{sd}=.0014)$. The effect size of Hedges' $\mathrm{g}=1.53$ implies a very large effect. While in Figure 4, the mean total of Auditory word score of those with low self-reported PTSD (M $=.0011, \mathrm{sd}=.0005)$ was statistically significantly different $(\mathrm{t}=-2.778, \mathrm{df}=38$, two-tailed $\mathrm{p}=.008)$ from that of people with high scores of self-reported PTSD $(\mathrm{M}=.0017, \mathrm{sd}=.0006)$. The effect size of Hedges' $\mathrm{g}=1.15$ implies a large effect. LIWC was used to examine differences in "othering" through the use of singular pronouns such as 'I,' 'me,' and 'my'.

The mean total 'I', "me", and 'my" word score of those with low self-reported PTSD (M =5.808, sd =1.079) was statistically significantly different $(\mathrm{t}=-2.419, \mathrm{df}=38$, two-tailed $\mathrm{p}=.02)$ from that of people with high scores of self-reported PTSD $(M=7.099, \mathrm{sd}=2.048)$. The effect size of Hedges' $g=-0.987$ implies a large effect. This was achieved through an independent-samples $t$-test and found significant differences existed in the linguistic characteristics between those deployed women who self-reported mental health concerns from those deployed women who did not self-report mental health concerns.

\section{DISCUSSION}

A range of statistically significant findings, summarised here, supported the hypothesis that the language from the military women differed to the language of the non-military women. In particular, deployed ADF mothers used less unique words, or more repetition in speech. This style of language is often seen in individuals with anxiety and depression due to a reduction in the ability to process language efficiently This was demonstrated through a lower Richness mean, a higher Referential Activity Power, and a lower sensory component mean score, which suggested that their language was more abstract and they relied less on stored imagery compared to the nonmilitary women's sample. The language and cognitive processing of the deployed women appears blunted with increasing years of service and that their language draws significantly on the expression of their lived memories. There is evidence of psychological distancing supported through the use of the word 'I' and a higher Referential Activity Power, and cognitive blunting as supported by sensory numbing in ADF mothers.

Given these statistically significant differences between the military women and the non-military women around Richness, Referential Activity Power, and Sensory component, we can plot a simplified Cognitive Processing (see Figure 5 top panel), where Referential Activity Power is higher and Richness lower than the control group. A significant inverse linear relationship was observed between Richness and Referential Activity Power, where Referential activity refers to the linking of an experience to the words used to express that experience. When there are high levels of Referential Activity Power, the speaker is showing a deep and vivid connection to the memory, recalling the emotions and sensations of the experience. When Referential Activity Power is low, language is more abstract, lacking detail and uses less imagery (The Referential Process (2020). Richness refers to "the number of unique words used by an author [or speaker]" (Bradbury, Bossomaier, \& Kernot, 2017). Richness is typically in a linear relationship with Referential Activity Power. This inverse linear relationship here highlights the blunting in the language and suggests that the deployed mothers are using higher imageability words and words with higher concreteness, in other words less abstract, and drawing on lived memories, but as can be seen, the cluster is quite tight and lower, suggesting word use is constrained from cognitive blunting.

As seen in the Sensory processing (see Figure 5 middle panel), Richness is low and sensory word use is also low. We would expect higher Sensory results given the higher Referential Activity Power results in the top panel of Figure 5, and so this would indicate that the language is constrained through sensory numbing. This is also reinforced by the cognitive blunting in the top panel of the cognitive processing figure.

Embodied cognition (see Figure 5 bottom panel) draws on the external world around us fed via our senses, and from the way we draw on lived experiences. In the Embodied Cognition panel, we see another tight cluster where Referential Activity is high, suggesting the language draws on lived experiences (has a ring of truth and sincerity about it) but is low in sensory aspects. This cognitive and sensory blunting is reflected in the top and middle panel figures.

There are also differences in the language used by female military personnel who report more mental health symptoms than those with few or no symptoms. Those with mental health conditions used words that focussed on the past and used fewer 'they' type words to effectively isolate themselves from those ADF personnel who were not suffering from mental health issues to minimise stigma. This type of language implies that female military personnel with mental health symptoms felt isolated from their peers, as though they were members of an outgroup. This term used to describe this feeling is "othering" through 'in-group' language versus the 'out-group', and suggests that women in this category feel stigmatised. This psychological distancing can be seen in singular pronouns use such as 'I,' 'me,' and 'my' (Nook, Schleider, \& Somerville, 2017) and observed in the linguistic 
characteristics between those deployed women who self-reported mental health concerns from those deployed women who did not self-report mental health concerns.

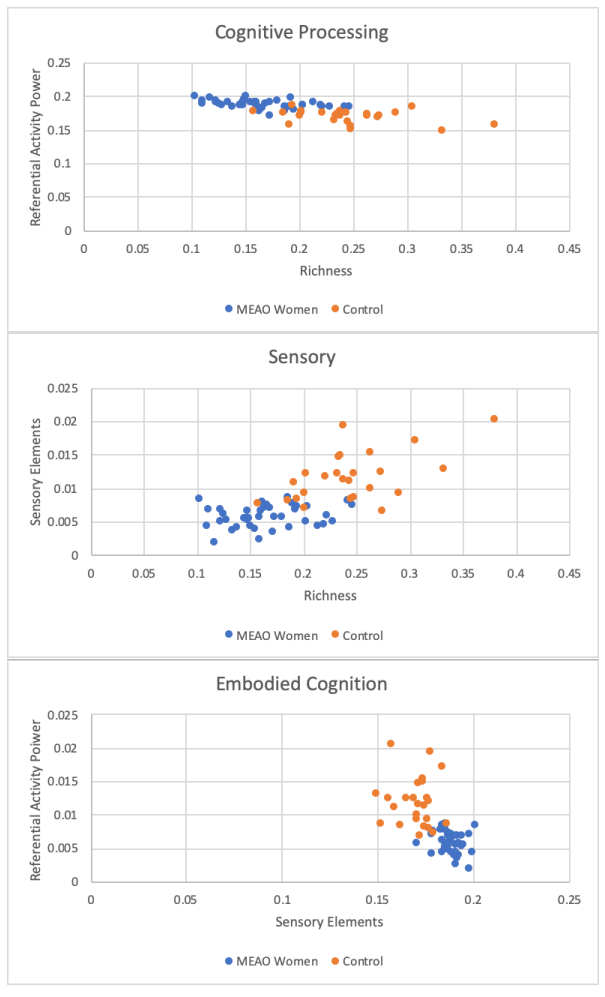

Figure 5. The statistically significant psycholinguistic variables are shown as three separately combined plots here from the RPAS algorithms (Kernot, 2019), where Richness (R), Referential Activity Power (A), and the five combined Sensory Elements (S) are used to show differences in the stylistic properties of both groups. Personal pronouns (P) that describe someone's internal Gender were not used directly within this overall panel directly, but indirectly are part of the Referential Activity Power variable.

Richness and Referential Activity Power are plotted as Cognitive Processing in the top pane. The middle pane shows the five senses Visual, Auditory, Haptic (touch and feel), Olfactory, and Gustatory are combined and represent all of the senses. Embodied Cognition in the bottom pane, as a result of cognitive processing and sensory input from stored memories highlights the less rich (more constrained with a number in that cohort more constrained than their peers hinting at difficulties in cognitive processing) less use of recalled sensory memories and tighter and slightly higher Referential Activity power measurement.

\subsection{Limitations}

This exploratory study was limited to 40 Australian military mothers deployed for a duration of 2-20 months.

\section{CONCLUSION}

This study supports the idea that linguistic analysis using embodied cognition can discriminate between military females with self-reported mental health and those without. Proposed future work includes deployed military men and emergency services first responders. These techniques might be a value aid in highlighting post-traumatic stress in veterans and first responders early in its development.

\section{ETHICS}

This research received ethics approval from Department of Defence and Veterans' Affairs Human Research Ethics Committee (DDVA HREC) ID 125-19. The Military and Veterans' Health Research Data Access Committee (MVHRDAC) approved access to the Mothers in the MEAO study (Lawrence-Wood et al., 2014) qualitative interview data (transcriptions) and all associated self-reported questionnaire data, ID MVHRD0042.

\section{ACKNOWLEDGMENTS}

We acknowledge the funding provided by the Defence Innovation Partnership (DIP) team, Andrew Fullgrabe, Dr Robin Nicholson, Sumen Rai and Lisa Paddick, via the South Australia Government and Defence Science and Technology Group. The assistance of Ms Karen May, The Road Home (now Military and Emergency Services Health Australia) and The Hospital Research Foundation in the initial phases of the research is acknowledged and appreciated. The research team acknowledges the Centre for Traumatic Stress Studies at the University of Adelaide and its researchers for the sharing of data from its study, Mothers in the Middle East Area of Operations (MEAO): the health impacts of maternal deployment to an area of operations, and we sincerely thank the former and serving military women who shared their stories. 


\section{REFERENCES}

Alorainy, W., Burnap, P., Liu, H. \& Williams, M.L. (2019). "'The enemy among us": Detecting cyber hate speech with threats-based othering language embeddings', ACM Transactions on the Web (TWEB), 13(3), pp. 1-26.

Alvarez-Conrad, J., Zoellner, L.A. \& Foa, E.B. (2001). 'Linguistic predictors of trauma pathology and physical health', Applied Cognitive Psychology, 15(7), pp. S159-S170.

American Psychiatric Association, D. S., \& American Psychiatric Association. (2013). Diagnostic and statistical manual of mental disorders: DSM-5.

Bradbury, R., Bossomaier, T. \& Kernot, D. (2017). 'Predicting the emergence of self-radicalisation through social media: A complex systems approach', in M Conway, L Jarvis, O Lehane, S Macdonald \& L Nouri (eds.), Terrorists' Use of The Internet, IOS Press, p. 381.

Bucci, W. \& Miller, N.E. (1993), 'Primary process analogue: The referential activity (RA) measure', in NE Miller, L Luborsky, JP Barber \& JP Docherty (eds.), Psychodynamic Treatment Research: A Handbook for Clinical Practice, Basic Books, New York, pp. 387-406.

Coppersmith, G., Harman, C. \& Dredze, M. (2014). 'Measuring post-traumatic stress disorder in Twitter', Eighth international AAAI Conference on Weblogs and Social Media, pp. 579-582.

Doyle, J. K., \& Ford, D. N. (1998). Mental models concepts for system dynamics research. System dynamics review: the journal of the System Dynamics Society, 14(1), 3-29.

Forrester, J. W. (1971). Counterintuitive behavior of social systems. Theory and decision, 2(2), 109-140.

Forrester, J. W. (1997). Industrial dynamics. Journal of the Operational Research Society, 48(10), 1037-1041.

Glenny, L., Kernot, D., Snowden, C., Van Hooff, M., Lawrence-Wood, E., Green, K., Son, C. (2020). Stigma in the Military: Identifying stigma through the language of a deployed Australian military population. Defence Innovation Partnership report.

Hotton, S., \& Yoshimi, J. (2010). The dynamics of embodied cognition. International Journal of Bifurcation and Chaos, 20(04), 943-972.

Hotton, S., \& Yoshimi, J. (2011). Extending dynamical systems theory to model embodied cognition. Cognitive Science, 35(3), 444-479.

Jaramillo, C.A., Eapen, B.C., McGeary, C.A., McGeary, D.D., Robinson, J., Amuan, M. \& Jo Pugh, M. (2016). 'A cohort study examining headaches among veterans of Iraq and Afghanistan wars: Associations with traumatic brain injury, PTSD, and depression', Headache: The Journal of Head and Face Pain, 56(3), pp. 528-539.

Kernot, D. (2019). 'An embodied cognition classifier of human emotion', in S. Eldswah (ed.), 23rd International Congress on Modelling and Simulation, Canberra, ACT. 1-6 December 2019, <http://mssanz.org.au/modsim2019/>.

Lawrence-Wood, E., Jones, L., Hodson, S., Crompvoets, S., McFarlane, A. \& Neuhaus, S. (2014). Mothers in the Middle East Area of Operations (MEAO): The Health Impacts of Maternal Deployment to an Area of Operations: Final Report, Centre for Traumatic Stress Studies, University of Adelaide, Adelaide.

Meadows, D. L., Behrens, W. W., Meadows, D. H., Naill, R. F., Randers, J., \& Zahn, E. (1974). Dynamics of growth in a finite world (p. 637). Cambridge, MA: Wright-Allen Press.

Murphy, S.M., Maskit, B. \& Bucci, W. (2015), 'Putting feelings into words: Cross-linguistic markers of the referential process', Proceedings of the Second Workshop on Computational Linguistics and Clinical Psychology: From Linguistic Signal to Clinical Reality, Denver, Colorado, pp. 80-88, <https://docplayer.net/104210447-Puttingfeelings-into-words-cross-linguistic-markers-of-the-referential-process.html $>$.

Nook, E.C., Schleider, J.L. \& Somerville, L.H. (2017). 'A linguistic signature of psychological distancing in emotion regulation', Journal of Experimental Psychology: General, 146(3), p. 337.

Papini, S., Yoon, P., Rubin, M., Lopez-Castro, T. \& Hien, D.A. (2015). 'Linguistic characteristics in a non-traumarelated narrative task are associated with PTSD diagnosis and symptom severity', Psychological Trauma: Theory, Research, Practice, and Policy, 7(3), pp. 295-302.

Paulin, J., Andersson, L. \& Nordin, S. (2016). 'Characteristics of hyperacusis in the general population', Noise \& Health, 18(83), p. 178.

Pennebaker Conglomerates, Inc. (2015). How it Works, viewed 22 January 2020, <http://liwc.wpengine.com/how-itworks/>.Port, R. F., \& Van Gelder, T. (Eds.). (1995). Mind as motion: Explorations in the dynamics of cognition. MIT press.

Pennebaker, J.W., Boyd, R.L., Jordan, K. \& Blackburn, K. (2015,). The Development and Psychometric Properties of LIWC 2015, The University of Texas at Austin, Austin, Texas.

Schöner, G., \& Reimann, H. (2019). Understanding Embodied Cognition through Dynamical System Thinking. In The Routledge Companion to Philosophy of Psychology (pp. 505-528). Routledge.

Smith, L. B. (2005). Cognition as a dynamic system: Principles from embodiment. Developmental Review, 25(3-4), 278-298.

Tausczik, YR \& Pennebaker, JW 2010, 'The psychological meaning of words: LIWC and computerised text analysis methods', Journal of Language and Social Psychology, 29(1), pp. 24-54.

The Referential Process (2020). Referential Activity, viewed 25 February 2020, $<\mathrm{http}$ ://www.thereferentialprocess.org/theory/referential-activity>.Wilson, A. D., \& Golonka, S. (2013). Embodied cognition is not what you think it is. Frontiers in psychology, 4, 58. 